\title{
HEAD AND NECK WARMING APPLIED IN THERMONEUTRAL CONDITIONS IMPROVES THERMAL SENSATION BUT DOES NOT ALTER COGNITIVE FUNCTION
}

\author{
Henrikas Paulauskas, Dalia Mickevičienè, Vaida Berneckè, \\ Kazimieras Pukẻnas, Marius Brazaitis \\ Institute of Sport Science and Innovations, Lithuanian Sports University, Kaunas, Lithuania
}

\begin{abstract}
Background. Heat therapy is widely used for therapeutic purposes in health SPA resorts or hospitals. However, its benefits and consequences are not strongly evidenced and it is partially-related with a lack of experimental information. No one has explored how continuous head and neck warming applied in thermoneutral environment can affect body temperatures, subjective sensations and cognition and the purpose of this study was to investigate it.

Methods. Seven subjects $(21 \pm 1$ years $)$ participated in two experimental trials: CON - control trial; and CONW - head and neck warming trial. These trials were designed to introduce the effects of continuous head and neck warming (surface warming by $44 \pm 1^{\circ} \mathrm{C}$ ) on thermal sensations, body temperatures and cognitive function in thermoneutral environment $\left(24^{\circ} \mathrm{C}\right.$ and $60 \%$ relative humidity). In each trial body temperatures (rectal, Tre; mean skin, Tsk), heart rate (HR) response, ratios of subjective sensations (SS) and cognitive function were assessed.

Results. Head and neck warming (CONW trial) maintained significantly higher Tre and Tsk values (by $0.2 \pm$ $0.1^{\circ} \mathrm{C}$ and $1 \pm 0.3{ }^{\circ} \mathrm{C}$, respectively), compared to the control (CON) trial. Head and neck warming caused significant increment in heart rate $(8 \pm 2 \mathrm{~b} / \mathrm{min})$ and improved thermal sensation $(p<.05)$, but had no effect on shivering/ sweating sensation and thermal comfort. Moreover, head and neck warming (CONW trial) does not induce any significant impact on accuracy $(p>.05)$ and response times $(p>.05)$ in mathematical and spatial processing tasks.

Conclusions. Continuous head and neck warming applied in thermoneutral conditions maintains higher internal (Tre) and peripheral (Tsk) body temperatures and improves thermal sensation, but does not alter cognitive function.
\end{abstract}

Keywords: head and neck warming, subjective sensations, body temperature, cognitive function.

\section{INTRODUCTION}

$\mathrm{H}$ eat therapy is widely used for therapeutic purposes in health SPA resorts and hospitals. The heat can be applied with hot water bottles, neck wraps, hot tubs, electric heating pads, etc. These various methods are certainly one of the frequently recommended non-invasive modalities for various kinds of acute or chronic pain relief (Garra et al., 2010; Malanga, Yan, \& Stark, 2015; Nadler et al., 2002; Nadler, Weingand, \& Kruse, 2004). Some residual after-effects associated with sports injuries, surgical procedures, broken bones can be minimized by using heat to increase blood circulation into the region, to help decrease bruising, inflammation, muscle spasms, swelling, stiffness as well as pain (Malanga et al., 2015; Nadler et al., 2004). However, it remains unclear whether local warming (usually used in heat therapy) induced changes in body temperatures and subjective sensations may affect cognitive function.

Inconsistent findings of previous investigators have shown that increment in both peripheral and core body temperatures may have negative (Gaoua, Grantham, Racinais, \& El Massioui, 2012; Solianik, Skurvydas, Mickevičienè, \& Brazaitis, 
2014; Sun et al., 2012), positive (Brazaitis et al., 2016) or no impact on cognitive function (Brazaitis et al., 2015). These inconsistent findings are a due to the large number of factors that come into play, such as task type, exposure duration, skill and acclimatization level of the individual and due to the absence of a concise theory on which experimental work can be based (Hancock \& Vasmatzidis, 2003; Taylor, Watkins, Marshall, Dascombe, \& Foster, 2016). Some previous reviews suggest that cognitive function is generally unaffected unless the external stimulus is sufficient in intensity and duration to increase core temperature away from a homeostatic range approximate to $37^{\circ} \mathrm{C}$ (Hancock \& Vasmatzidis, 2003). However, recent studies demonstrates that an increase in Tsk, independent of any rise (Gaoua et al., 2012) or decline (Brazaitis et al., 2016) in core temperature, may be responsible for alterations in cognitive function.

The head, neck, and face are regions of high allesthesial thermosensitivity (Cotter \& Taylor, 2005) and investigators have proposed that the neck might be an optimal site for temperature manipulations because of its close proximity to the thermoregulation centre (Gordon, Bogdanffy, \& Wilkinson, 1990; Shvartz, 1976). The previous studies have demonstrated that cooling the head and neck as compared to cooling equal body surface area on the torso, arm and thighs is more efficient at dissipating heat, reducing thermal strain and improving thermal comfort (Nunneley \& Maldonado, 1983; Shvartz, 1970, 1976). Furthermore, head and neck cooling applied in hyperthermic conditions may mask the true state of the body (Tyler \& Sunderland, 2011a, 2011b), alleviate unpleasant sensation (Cohen, Allan, \& Sowood, 1989; Nunneley \& Maldonado, 1983) decrease heart rate, oxygen consumption (Watanuki, 1993), and allow to tolerate higher rectal temperatures (Tyler \& Sunderland, 2011a).

The aforementioned studies demonstrate that the head and neck cooling effects are quite well established. However, the physiological and psychological head and neck warming effects have not been investigated and remain unknown. Recent research suggests that increased skin temperature (Tsk), independent of any rise in core temperature, may be responsible for any heat induced cognitive deteriorations (Gaoua et al., 2012). Thus, we hypothesized that continuous head and neck warming (surface warming by $44 \pm 1^{\circ} \mathrm{C}$ ) applied in thermoneutral environment should affect subjective sensations and cognitive function without affecting internal (Tre) body temperature.

\section{METHODS}

Participants. Seven healthy male volunteers (age $21 \pm 1$ year, height $181 \pm 2.4 \mathrm{~cm}$, weight $81.9 \pm 6.0 \mathrm{~kg}$, body mass index $24.8 \pm 1.3$, body fat $17.0 \pm 2.1 \%$, and body surface area $2.0 \pm 0.1 \mathrm{~m}^{2}$ ) participated in the study which was approved by LUHS Kaunas Region Biomedical Research Ethics Committee and conducted according to the guidelines of the Declaration of Helsinki. The participants were moderately physically active $(<3$ times per week) and did not participate in any formal physical exercise, sports program and were not involved in any temperature-manipulation programme or procedure for at least 1 year. The additional criteria for inclusion were age 18-30 years, being non-smokers, and not taking medication or dietary supplements.

Research design. The research design is presented in Figure 1. One week before starting the experiment, each subject read and signed a written informed consent form, and was familiarized with the experimental procedures for the subjective scales and cognitive testing. The software used for the cognitive testing battery (see "Cognitive function assessment") provided a familiarization procedure for each test before completing the full test procedure of both cognitive tests.

During the experiment, participants attended the laboratory at the same time of day within the time frame of 8:00-13:00 h to avoid circadian fluctuations in body temperature. They were instructed to sleep for a minimum $7-8 \mathrm{~h}$ on the night before the experiment; to refrain from alcohol, heavy exercise, and caffeine for at least $24 \mathrm{~h}$; and to refrain from consuming any food for at least $12 \mathrm{~h}$ before arrival at the laboratory. To standardize the morning state of hydration, subjects were allowed to drink still water as desired until $60 \mathrm{~min}$ before the experiment. The study was performed at a room temperature of $24^{\circ} \mathrm{C}$ and $60 \%$ relative humidity.

Experiment consisted of two experimental trials: CON trial (included lying in relax chair in a semi-recumbent 1posture for 170 minutes and cognitive function testing) and CONW trial (included lying in relax chair in a semi-recumbent posture for 170 minutes wearing head and neck warming device and cognitive function assessment 
wearing head and neck warming device). Each trial lasted approximately 175 min (170 minutes of lying in relax chair + cognitive function testing). Experimental trials were randomized and separated by one week break. All measurements and experimental procedures in these trials were identical (Figure 1).

On arrival at the laboratory the participant was asked to prepare for the resting in a semi-recumbent posture for 20 minutes dressed in swimming shorts. During these 20 minutes of resting, heart rate (HR), rectal (Tre) and skin (Tsk) temperature stabilization was assessed and recorded. Within $\sim 3$ minutes after these measurements participants were asked to assess subjective sensations (SS) and one of experimental trial (CON or CONW) started. Before lying in relax chair in the CONW trial, head and neck warming device (Figure 2) was fitted to the head and neck and kept until the end of the trial. Throughout each experimental trial subjective sensations (thermal sensation and shivering/sweating sensation), HR, Tre, were recorded every 5 minutes. Final HR, Tre, Tsk and ratings of subjective perception were recorded at the end of each trial (Figure 1).

Measurements. During the initial visit the height $(\mathrm{cm})$ of participants was measured, and body mass $(\mathrm{kg})$, body fat (\%), body mass index were determined by taking their nude body mass with body composition analyser (Tanita, TBF-300, Illinois, USA).

HR was measured at rest and throughout each experimental trial using a HR monitor (S-625X, Polar Electro, Kempele, Finland).

Tre was measured at rest and throughout each experimental trial using a thermocouple (Rectal Probe; Ellab, Hvidovre, Denmark; accuracy \pm $0.01^{\circ} \mathrm{C}$ ) inserted to a depth of $12 \mathrm{~cm}$ past the anal sphincter. The rectal thermistor sensor was placed by each participant.

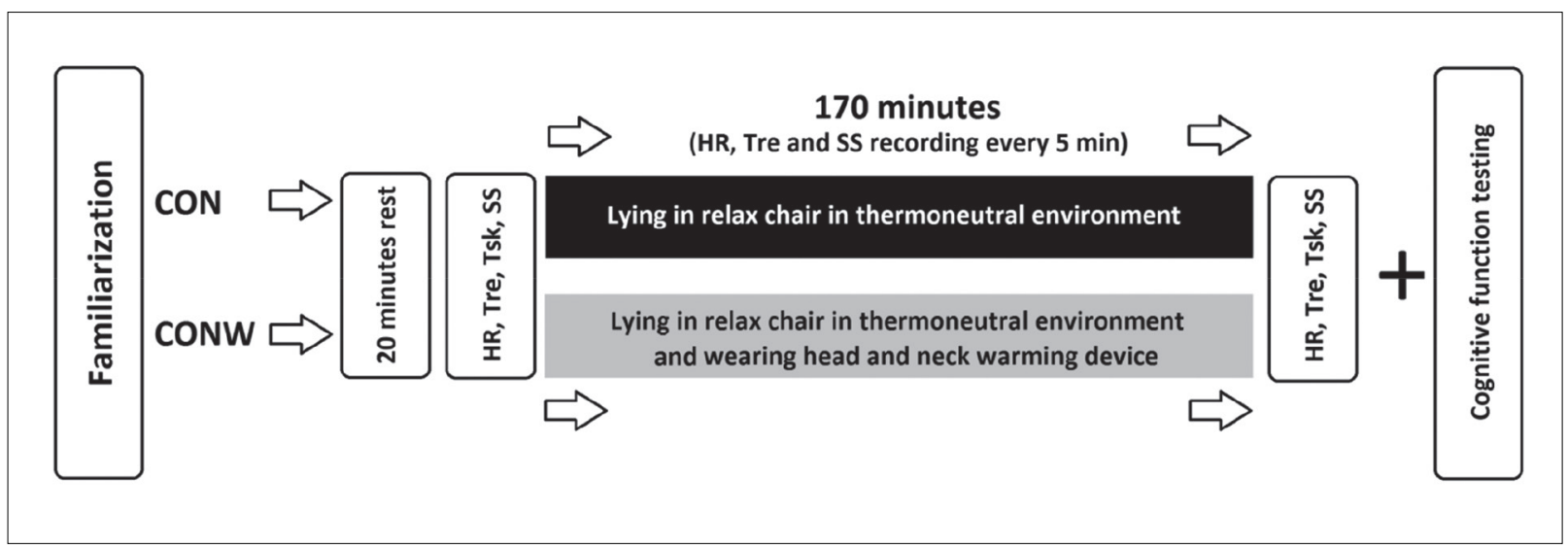

Figure 1. Research design

Notes. CON and CONW - experimental trials. Measurements of heart rate (HR) and body temperatures (rectal, Tre; skin, Tsk). Thermal sensation, thermal comfort and shivering/sweating sensation assessment (SS).

A

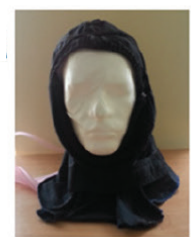

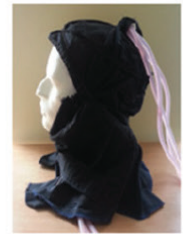

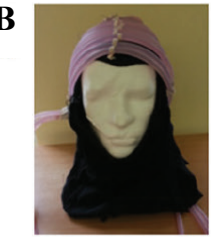

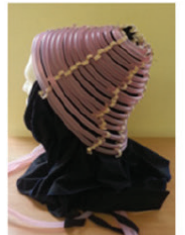
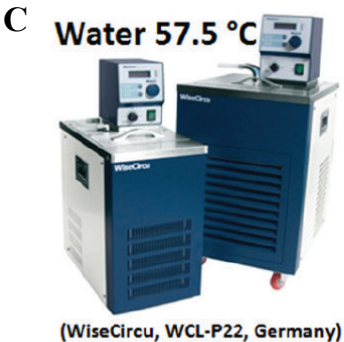

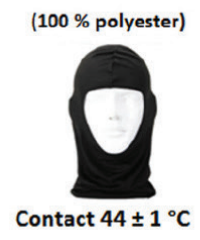

$\left(\begin{array}{c}\text { head and neck with } \\ \text { underhelmet }\end{array}\right)$

Figure 2. Head and neck warming device

Notes. A - outer view of head-neck device, B - inner view of head-neck device, C - example of circulator and underhelmet. 
Tsk was measured with thermistors (Skin/ Surface probe, DM852, Ellab; accuracy $\pm 0.01^{\circ} \mathrm{C}$ ) taped at three sites: back, thigh and forearm. The mean Tsk was calculated using the Burton (1935) equation: Tsk $=0.5_{\text {back }}+0.36_{\text {thigh }}+0.14_{\text {forearm }}$. Tsk temperature measurements were taken before and after each experimental trial.

Rating of subjective sensations. The method to measure subjective ratings for the whole body has been described elsewhere (Brazaitis et al., 2014; Brazaitis, Kamandulis, Skurvydas, \& Daniuseviciute, 2010). Briefly, ratings of thermal sensation ranged between 1 (very cold) to 9 (very hot), with 5 being neutral. The shivering/sweating sensation ranged from 1 (vigorously shivering) to 7 (heavily sweating), with 4 (not at all) being neutral. Thermal comfort sensation ranged from 1 (comfortable) to 5 (extremely uncomfortable). The rating of subjective sensations was reported by the participant after resting and every 5 min during each trial. The mean rating score value per trial was calculated.

Cognitive function assessment. Automated Neuropsychological Assessment Metric (ANAM 4 , Norman, OK, USA) was administered (Reeves, Kane, Winter, Raynsford, \& Pancella, 2010). All tests were computer (Samsung R538) controlled with the information being presented on the screen of a laptop ( $\sim 40 \mathrm{~cm}$ in front of the participant). Cognitive tests battery was performed in a quiet and semi-darkened laboratory. The duration for performing the ANAM test battery was $2 \pm$ $0.5 \mathrm{~min}$ and included the following tasks which, were randomized in the $(\mathrm{CON}, \mathrm{CONW})$ trials. Cognitive test battery was performed at the end of each experimental trial, immediately after resting in relax chair (see 'Research Design'). In each cognitive test the percentage of accurate response and response time were determined.

Mathematical Processing. Results of this test are used as an index of basic computational skills, concentration, and working memory (Kabat, Kane, Jefferson, \& DiPino, 2001). During this task, an arithmetic problem involving three single-digit numbers and two operators is displayed (e.g. 5 $2+3=$ ). The user presses buttons to indicate whether the answer to the problem is less than five or greater than five.

Spatial Processing. This test presents two four-bar histograms, the first of which is displayed upright and the second of which is displayed after a 90 degree rotation either clockwise or counter-clockwise. The user presses designated buttons to indicate if the two histograms are the same or different, regardless of the orientation. This test relies heavily on visual spatial skills and mental rotation (Reeves et al., 2010). The examinee must be able to perceive the histograms accurately and determine if they match after mentally rotating one of them. Because this task is based on speed and accuracy of performance it also requires parallel cognitive processes of sustained and selective attention and information processing speed.

Head and neck warming device. The system was designed using circulator (WiseCircu, WCL-P22, Germany), silicone tubes and firefighter underhelmet made of fire-resistant material (Figure 2). The pilot study was performed to determine maximal available temperature for warming without discomfort (burning sensation); temperature changes in different contact places; and temperature stabilization time after putting the head-neck device. The temperature between frontal, dorsal and lateral contact places of the head and dorsal contact place of the neck wearing headneck device with underhelmet (100\% polyester) varied in $44 \pm 1^{\circ} \mathrm{C}$ range during 3 hours of warming procedure (contact place: head and/or neck with underhelmet temperature was measured with Skin/Surface probe, DM852, Ellab). Temperature stabilization in the contact places was reached in 5 minutes. To keep this temperature in $44 \pm 1{ }^{\circ} \mathrm{C}$ range the circulator's liquid (water) temperature was raised and maintained in $57.5^{\circ} \mathrm{C}$.

Statistical analysis. Statistical analyses were performed using IBM SPSS Statistics software (v. 22; IBM Corp., Armonk, NY). The data of experiment were tested for normal distribution using the Kolmogorov-Smirnov test, and all data were found to be normally distributed. Descriptive data are presented as mean \pm standard error (SE) of the mean. A two-factor (trial (CON vs. $\mathrm{CONW}) \times$ time (before vs. after)) repeatedmeasures analysis of variance (ANOVA) was used to analyse the changes in thermophysiological responses (Tre, $\Delta$ Tre, Tsk, $\Delta$ Tsk, HR, $\Delta \mathrm{HR}$ ) and cognition (response time, and accuracy of cognitive tests). If significant effects were found, Sidak's post hoc adjustment was used to locate the difference. Statistical significance was defined as $p>.05$. Statistical power ( $S P$, as percentage) was calculated, and the partial eta squared $\left(\eta_{p}{ }^{2}\right)$ was estimated as a measure of time or trial effect size. The non-parametric Wilcoxon signed-rank test for 
two related samples was used to compare changes in ratings of subjective sensations (thermal and shivering/sweating sensations) between $\mathrm{CON}$ and CONW trials.

\section{RESULTS}

Lying in relax chair lasted for the same amount of time $(170 \mathrm{~min})$ in both experimental trials (CON and $\mathrm{CONW}$ ). There were no significant differences between HR, body temperature values (Tre, Tsk) $\left(\mathrm{CON}\right.$ vs. CONW trial effect; $p>.05, \eta_{p}{ }^{2}<0.19, S P<$ $17 \%)$ and rating of subjective sensations $(p>.05)$ measured before the experimental trials (Table 1).

Tre did not change significantly in experimental (CON and CONW) trials. However, Tre remained significantly higher (by $0.2^{\circ} \mathrm{C}$ ) after lying in relax chair with head and neck warming device (CONW trial), compared with CON trial (CON vs. CONW time effect; $p<.05, \eta_{p}{ }^{2}=0.6$, $S P=80 \%)$. The change in Tre also differed significantly between experimental trials $(\triangle \mathrm{CON}$ vs. $\triangle \mathrm{CONW}$ trial effect; $p<.05, \eta_{p}{ }^{2}=0.5, S P=$ $63 \%$ ). Tsk significantly decreased (by $0.9{ }^{\circ} \mathrm{C}$ ) after lying in relax chair in the CON trial (time effect in the CON trial; $p<.05, \eta_{p}{ }^{2}=0.77, S P=97 \%$ ), but did not change in the CONW trial. Thus, after the CONW trial Tsk was significantly higher (by $\left.1^{\circ} \mathrm{C}\right)$, comparing with $\mathrm{CON}$ trial $(\triangle \mathrm{CON}$ vs. $\triangle \mathrm{CONW}$ trial effect; $p<.05, \eta_{p}{ }^{2}=0.66$, $S P=80 \%$ ). HR did not change in the CON trial, but increased significantly when head and neck warming was applied (time effect in the CONW trial; $p<.05, \eta_{p}{ }^{2}=0.76, S P=95 \%$ ). Furthermore, the change in HR significantly differed between trials $(\triangle \mathrm{CON}$ vs. $\triangle \mathrm{CONW}$ trial effect; $p<.05$, $\eta_{p}{ }^{2}=0.59, S P=68 \%$ ) (Table 1$)$.

Table 2 summarizes subjective ratings of thermal sensation, thermal comfort and shivering/ sweating sensation in experimental trials. Subjects felt warmer $(p<.05)$ in the CONW trial, compared to $\mathrm{CON}$ trial. However, shivering/sweating sensation and thermal comfort did not differ between trials.

Figure 3 summarizes accuracy $(\%)$ and response time (ms) changes in the mathematical processing and spatial processing (simultaneous) tasks in experimental trials. There were no significant differences in accuracy (CON vs. CONW trial effect; $p>.05, \eta_{p}{ }^{2}<0.07, S P<9 \%$ ) and response times (CON vs. CONW trial effect; $p>.05, \eta_{p}{ }^{2}<$ $0.07, S P<9 \%$ ) in both cognitive tasks, comparing $\mathrm{CON}$ and $\mathrm{CONW}$ trials (Figure 3 ).

\begin{tabular}{|c|c|c|c|c|}
\hline & \multicolumn{2}{|c|}{ CON } & \multicolumn{2}{|c|}{ CONW } \\
\hline & Before & After & Before & After \\
\hline $\mathrm{HR}, \mathrm{b} / \mathrm{min}$ & $60 \pm 5$ & $57 \pm 3$ & $55 \pm 2$ & $64 \pm 3 * \#$ \\
\hline$\Delta$ & \multicolumn{2}{|c|}{$-3 \pm 3$} & \multicolumn{2}{|c|}{$8 \pm 2 \#$} \\
\hline$\Delta \Delta$ & \multicolumn{4}{|c|}{$11 \pm 4$} \\
\hline Tre, ${ }^{\circ} \mathrm{C}$ & $36.9 \pm 0.1$ & $36.8 \pm 0.1$ & $36.9 \pm 0.1$ & $37.0 \pm 0.1 \#$ \\
\hline$\Delta$ & \multicolumn{2}{|c|}{$-0.1 \pm 0.3$} & \multicolumn{2}{|c|}{$0.1 \pm 0.2 \#$} \\
\hline$\Delta \Delta$ & \multicolumn{4}{|c|}{$0.2 \pm 0.1$} \\
\hline Tsk, ${ }^{\circ} \mathrm{C}$ & $31.7 \pm 0.2$ & $30.8 \pm 0.3 *$ & $31.8 \pm 0.2$ & $31.9 \pm 0.2 \#$ \\
\hline$\Delta$ & \multicolumn{2}{|c|}{$-0.9 \pm 0.2$} & \multicolumn{2}{|c|}{$0.1 \pm 0.3 \#$} \\
\hline$\Delta \Delta$ & & & & \\
\hline
\end{tabular}

\begin{tabular}{|c|c|c|c|}
\hline & CON & CONW & \\
\hline $\begin{array}{c}\text { Sweeting/shivering } \\
\text { sensation }\end{array}$ & $4.0 \pm 0.0$ & $4.1 \pm 0.1$ & $\begin{array}{c}\text { Sweeting sensation did } \\
\text { not change }\end{array}$ \\
\hline Thermal sensation & $4.7 \pm 0.1$ & $5.7 \pm 0.3 \#$ & $\begin{array}{c}\text { Thermal sensation } \\
\text { improved }\end{array}$ \\
\hline Thermal comfort & $1.0 \pm 0.0$ & $1.0 \pm 0.0$ & $\begin{array}{c}\text { Thermal comfort did not } \\
\text { change }\end{array}$ \\
\hline
\end{tabular}

Table 1. Body temperatures and heart rate in the experimental (CON and CONW) trials
Notes. $\Delta-$ mean difference between before and after values, $\Delta \Delta-$ difference of mean difference between CON and CONW trials, ${ }^{*} p<.05$, compared to before, $\# p<.05$, compared to $\mathrm{CON}$. Values are means $\pm S E$.

Table 2. Sweating/shivering sensation and thermal sensation in the experimental (CON and CONW) trials

Notes. $\# p<.05$, compared to $\mathrm{CON}$ Values are means $\pm S E$. 


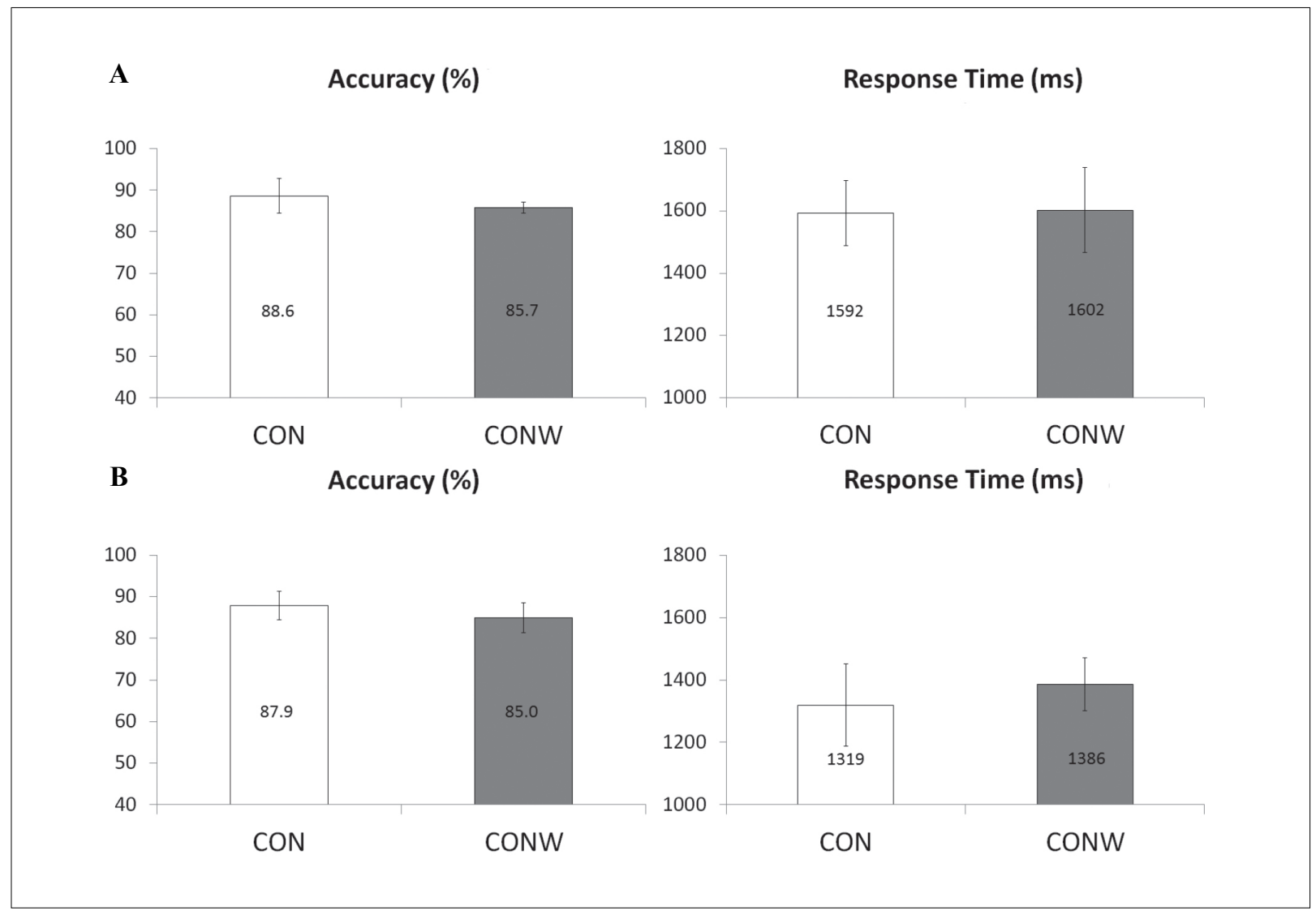

Figure 3. Accuracy (\%) and response times (milliseconds) in (A) mathematical processing and (B) spatial processing (simultaneous) tasks

\section{DISCUSSION}

Our expectations that continuous head and neck warming applied in a thermoneutral environment (CONW trial) should affect subjective sensations and cognitive function without affecting internal (Tre) body temperature were not confirmed. The findings in the study demonstrated that head and neck warming applied in thermoneutral conditions increased heart rate, maintained higher internal (Tre) and peripheral (Tsk) body temperatures and improved thermal sensation without alterations in cognitive function, thermal comfort and shivering/ sweating sensation.

Continuous head and neck warming applied in thermoneutral conditions during 170 minutes lying in relax chair (CONW trial) was sufficient to maintain higher Tre (by $0.2^{\circ} \mathrm{C}$ ) and mean Tsk (by $1{ }^{\circ} \mathrm{C}$ ), compared with CON trial (Table 1). We believe that higher body temperatures preserved in the head and neck warming trial may possibly be explained by heat transfer from head and neck warming device to superficial head and neck regions via conduction (constant $44 \pm 11^{\circ} \mathrm{Ctemperature),} \mathrm{and} \mathrm{laterheat} \mathrm{transfer}$ to internal organs and other peripheral body regions via blood circulation. In the head and neck warming trial (CONW), blood circulation was improved due to significant heart rate increment (Table 1) and this might ensure better heat transfer to other body regions. According to Nakamura and Morrison (2010), skin warming induced tachycardia would help the heat dissipating effect of the simultaneous cutaneous vasodilation by maintaining cardiac output and arterial pressure at a sufficient level to assure optimal increases in cutaneous blood flow. Heat transfer from one body region to another was also observed in previous studies where peripheral feet and lower legs' warming in $42^{\circ} \mathrm{C}$ water $(25$ and $35^{\circ} \mathrm{C}$ ambient conditions) was sufficient to increase Tre and Tsk (chest, back, forearm, forehead and thigh) in young subjects (Inoue \& Shibasaki, 1996; Inoue, Shibasaki, Hirata, \& Araki, 1998). Similarly, in a recent (Brazaitis et al., 2015) study it was demonstrated that immersion to the waistline in $43-44^{\circ} \mathrm{C}$ water markedly increased both internal (Tre by $\sim 2.5^{\circ} \mathrm{C}$ ) and peripheral (Tsk $\sim 4^{\circ} \mathrm{C}$ ) body temperatures. Besides, this study showed that even the vast increment in body temperatures may not induce any significant alterations in cognitive function. 
Despite controversial findings, it is almost universally accepted that various aspects of attention (Gaoua, Racinais, Grantham, \& El Massioui, 2011; Hancock, 1986; Simmons, Saxby, McGlone, \& Jones, 2008), memory (Gaoua et al., 2011; Jiang et al., 2013; Racinais, Gaoua, \& Grantham, 2008), and executive function (Gaoua et al., 2012; Gaoua et al., 2011) are reduced in heat-stressed persons. In the CONW trial, head and neck were constantly affected by $\sim 44^{\circ} \mathrm{C}$ temperature, but it was insufficient to induce heat stress increasing internal temperature away from a homeostatic range (Tre was $37 \pm 0.1^{\circ} \mathrm{C}$ ). Besides, head and neck warming (CONW trial) caused significant improvement in thermal sensation (from "neutral" to "slightly warm"), but did not induce any alterations in shivering/ sweating sensation (subjects did not feel shivering or sweating) and thermal comfort (subjects felt comfortable) (Table 2). According to Gaoua et al. (2012), the subjective state of the individual could be a key factor affecting cognitive function in the heat. The authors demonstrated that exposure to $50^{\circ} \mathrm{C}$ (for $\sim 15 \mathrm{~min}$ ) induced increment in mean Tsk $\left(\sim 3^{\circ} \mathrm{C}\right.$, without any change in core temperature and motor cortical excitability) and thus reduced thermal comfort, improved negative feelings (i.e. subjects felt hotter and less comfortable) and impaired complex (working memory) task performance, while it had no significant effect on simple task performance. In a current study, subjects felt slightly warm and comfortable, and this might be one of the reasons why mathematical and spatial processing tasks performance was not affected (Figure 3). The other reason might be rather simple cognitive tasks. It is generally accepted that in the hot conditions simple task performance is less vulnerable than complex task performance (Gaoua et al., 2012; Hancock, 1986; Pilcher, Nadler, \& Busch, 2002).

Similarly to the results found in our study, Watkins et al. (2014) established that slightly, but significantly higher Tre $\left(37.1 \pm 0.3^{\circ} \mathrm{C}\right)$, Tsk $(33.2 \pm$ $\left.1.1^{\circ} \mathrm{C}\right)$, increased heart rate $(82.1 \pm 13.9 \mathrm{~b} / \mathrm{min})$ and thermal sensation did not deteriorate soccer goal line officials' ability to complete simple tasks (tracking, simple reaction time, and numerical vigilance) during a $90 \mathrm{~min}$ passive exposure to $30^{\circ} \mathrm{C}$. In earlier report, Simmons et al. (2008) demonstrated that cognitive function remained stable even if whole body warming increased Tsk (by $\sim 2^{\circ} \mathrm{C}$, without increment in Tcore) and caused significant heart rate increment and thermal discomfort. However, in this study we used rather simple cognitive tasks. Very recent study reported that increasing thermal comfort (rather than mitigating Tcore increases) may be effective in maintaining complex cognitive function (and consequently safety) in passively experienced thermally stressful environments (Taylor et al., 2016). Moreover, selective head skin cooling, which alleviates unpleasant sensation in a heat (Cohen et al., 1989; Nunneley \& Maldonado, 1983), has been shown to preserve some complex cognitive functions during passive hyperthermia (Gaoua et al., 2011). Aforementioned studies support that cognitive decline (especially in a complex cognitive tasks) is primarily mediated by a reduction in thermal comfort. This is consistent with the distraction hypothesis, which suggests that discomfort caused by temperature stimulus may cause a shift of attention from the primary task and thus lead to impaired performance (Teichner, 1958).

In agreement with previous findings, a present study demonstrates that cognitive function may remain stable, mainly because of rather simple cognitive tasks and absence of heat stress and discomfort. Head and neck warming seems promising technique to maintain higher internal and peripheral body temperatures and improve thermal sensation for humans resting in normothermic conditions. The greater body area of peripheral warming should be considered to avoid internal temperature increment away from a homeostatic range. The current study focused on changes in body temperatures, heart rate, subjective sensations and specific cognitive (mathematical and spatial processing) tasks in normothermic conditions. Therefore, further research is needed to explore and validate possible head and neck warming effects on thermogenesis, neuromuscular function and cognition, especially in more extreme (ex. cold) environments.

\section{CONCLUSIONS}

Continuous head and neck warming applied in thermoneutral conditions maintain higher internal (Tre) and peripheral (Tsk) body temperatures and improves thermal sensation. However, these alterations are insufficient to induce any significant impact on accuracy and response times in mathematical and spatial processing tasks.

Acknowledgements. The authors acknowledge the subjects who took part in the study. 


\section{REFERENCES}

Brazaitis, M., Eimantas, N., Daniuseviciute, L., Mickeviciene, D., Steponaviciute, R., \& Skurvydas, A. (2014). Two Strategies for response to $14^{\circ} \mathrm{C}$ cold-water immersion: Is there a difference in the response of motor, cognitive, immune and stress markers? PloS One, 9(10), e109020-e109020. doi: 10.1371/journal.pone.0109020

Brazaitis, M., Eimantas, N., Daniuseviciute, L., Vitkauskiene, A., Paulauskas, H., \& Skurvydas, A. (2015). Two strategies for the acute response to cold exposure but one strategy for the response to heat stress. International Journal of Hyperthermia, 31(4), 325-335. doi: 10.3109/02656736.2015.1004135

Brazaitis, M., Kamandulis, S., Skurvydas, A., \& Daniuseviciute, L. (2010). The effect of two kinds of T-shirts on physiological and psychological thermal responses during exercise and recovery. Applied Ergonomics, 42(1), 46-51. doi: 10.1016/j. apergo.2010.04.001

Brazaitis, M., Paulauskas, H., Skurvydas, A., Budde, H., Daniuseviciute, L., \& Eimantas, N. (2016). Brief rewarming blunts hypothermia-induced alterations in sensation, motor drive and cognition. Frontiers in Physiology, 7(592). doi: 10.3389/fphys.2016.00592

Burton, A. C. (1935). Human calorimetry: The average temperature of the tissues of the body. Journal of Nutrition, 9, 261-280.

Cohen, J. B., Allan, J. R., \& Sowood, P. J. (1989). Effect of head or neck cooling used with a liquid-conditioned vest during simulated aircraft sorties. Aviation Space and Environmental Medicine, 60(4), 315-320.

Cotter, J. D., \& Taylor, N. A. S. (2005). The distribution of cutaneous sudomotor and alliesthesial thermosensitivity in mildly heat-stressed humans: An open-loop approach. The Journal of Physiology, 565(1), 335-345. doi: 10.1113/jphysiol.2004.081562

Gaoua, N., Grantham, J., Racinais, S., \& El Massioui, F. (2012). Sensory displeasure reduces complex cognitive performance in the heat. Journal of Environmental Psychology, 32(2), 158-163. doi: 10.1016/j. jenvp.2012.01.002

Gaoua, N., Racinais, S., Grantham, J., \& El Massioui, F. (2011). Alterations in cognitive performance during passive hyperthermia are task dependent. International Journal of Hyperthermia, 27(1), 1-9. doi: 10.3109/02656736.2010.516305

Garra, G., Singer, A. J., Leno, R., Taira, B. R., Gupta, N., Mathaikutty, B., \& Thode, H. J. (2010). Heat or cold packs for neck and back strain: A randomized controlled trial of efficacy. Academic Emergency Medicine, 17(5), 484-489. doi:10.1111/j.1553-2712.2010.00735.x

Gordon, N., Bogdanffy, G., \& Wilkinson, J. (1990). Effect of a practical neck cooling device on core temperature during exercise. Medicine and Science in Sports and Exercise, 22(2), 245-249.

Hancock, P. A. (1986). Sustained attention under thermal stress. Psychological Bulletin, 99(2), 263-281. doi:10.1037/0033-2909.99.2.263
Hancock, P. A., \& Vasmatzidis, I. (2003). Effects of heat stress on cognitive performance: The current state of knowledge. International Journal of Hyperthermia, 19(3), 355-372. doi: 10.1080/0265673021000054630

Inoue, Y., Shibasaki, M., Hirata, K., \& Araki, T. (1998). Relationship between skin blood flow and sweating rate, and age related regional differences. European Journal of Applied Physiology and Occupational Physiology, 79(1), 17-23. doi: 10.1007/s004210050467

Inoue, Y., \& Shibasaki, M. (1996). Regional differences in age-related decrements of the cutaneous vascular and sweating responses to passive heating. European Journal of Applied Physiology and Occupational Physiology, 74(1), 78-84. doi: 10.1007/bf00376498

Jiang, Q., Yang, X., Liu, K., Li, B., Li, L., Li, M., . . . Sun, G. (2013). Hyperthermia impaired human visual short-term memory: An fMRI study. International Journal of Hyperthermia, 29(3), 219-224. doi: 10.3109/02656736.2013.786141

Kabat, M. H., Kane, R. L., Jefferson,A. L., \& DiPino, R. K. (2001). Construct validity of Selected Automated Neuropsychological Assessment Metrics (ANAM) battery measures. The Clinical Neuropsychologist, 15(4), 498-507. doi:10.1076/clin.15.4.498.1882

Malanga, G. A., Yan, N., \& Stark, J. (2015). Mechanisms and efficacy of heat and cold therapies for musculoskeletal injury. Postgraduate Medicine, 127(1), 57-65. doi: 10.1080/00325481.2015.992719

Nadler, S. F., Steiner, D. J., Erasala, G. N., Hengehold, D. A., Hinkle, R. T., Goodale, M. B., . . . Weingand, K. W. (2002). Continuous low-level heat wrap therapy provides more efficacy than ibuprofen and acetaminophen for acute low back pain. Spine, 27(10), 1012-1017. doi:10.1097/00007632-200205150-00003

Nadler, S. F., Weingand, K., \& Kruse, R. J. (2004). The physiologic basis and clinical applications of cryotherapy and thermotherapy for the pain practitioner. Pain Physician, 7(3), 395-400. Retrieved from http://www. preventworkinjury.com/wp-content/uploads/2014/10/ Heat-vs-Cold-Therapy.pdf

Nakamura, K., \& Morrison, S. F. (2010). A thermosensory pathway mediating heat-defense responses. Proceedings of the National Academy of Sciences of the United States of America, 107(19), 8848-8853. doi: 10.1073/ pnas.0913358107

Nunneley, S., \& Maldonado, R. (1983). Head and/ or torso cooling during simulated cockpit heat stress. Aviation, Space, and Environmental Medicine, 54(6), 496-499.

Pilcher, J. J., Nadler, E., \& Busch, C. (2002). Effects of hot and cold temperature exposure on performance: A meta-analytic review. Ergonomics, 45(10), 682-698. doi: 10.1080/00140130210158419

Racinais, S., Gaoua, N., \& Grantham, J. (2008). Hyperthermia impairs short-term memory and peripheral motor drive transmission. The Journal of Physiology, 586(19), 4751-4762. doi: 10.1113/jphysiol.2008.157420 
Reeves, D., Kane, R., Winter, K., Raynsford, K., \& Pancella, T. (2010). ANAM4 ${ }^{\mathrm{TM}}$ Sports Medicine Battery. Automated neuropsychological assessment metrics (Version 4). Norman, OK, USA: Center for the Study of Human Operator Performance.

Shvartz, E. (1970). Effect of a cooling hood on physiological responses to work in a hot environment. Journal of Applied Physiology, 29(1), 36-39. Retrieved from http://jap.physiology.org/content/jap/29/1/36.full.pdf Shvartz, E. (1976). Effect of neck versus chest cooling on responses to work in heat. Journal of Applied Physiology, 40(5), 668-672. Retrieved from http://jap. physiology.org/content/jap/40/5/668.full.pdf

Simmons, S. E., Saxby, B. K., McGlone, F. P., \& Jones, D. A. (2008). The effect of passive heating and head cooling on perception, cardiovascular function and cognitive performance in the heat. European Journal of Applied Physiology, 104(2), 271-280. doi: 10.1007/ s00421-008-0677-y

Solianik, R., Skurvydas, A., Mickevičienè, D., \& Brazaitis, M. (2014). Intermittent whole-body cold immersion induces similar thermal stress but different motor and cognitive responses between males and females. Cryobiology, 69(2), 323-332. doi: 10.1016/j. cryobiol.2014.08.007

Sun, G., Yang, X., Jiang, Q., Liu, K., Li, B., Li, L., . . . Li, M. (2012). Hyperthermia impairs the executive function using the Attention Network Test. International
Journal of Hyperthermia, 28(7), 621-626. doi: 10.3109/02656736.2012.705217

Taylor, L., Watkins, S. L., Marshall, H., Dascombe, B. J., \& Foster, J. (2016). The impact of different environmental conditions on cognitive function: A focused review. Frontiers in Physiology, 6(372). doi: 10.3389/fphys.2015.00372

Teichner, W. H. (1958). Reaction time in the cold. Journal of Applied Psychology, 42(1), 54-59. doi: 10.1037/h0049145

Tyler, C. J., \& Sunderland, C. (2011a). Cooling the neck region during exercise in the heat. Journal of Athletic Training, 46(1), 61-68. doi: 10.4085/1062-6050-46.1.61

Tyler, C. J., \& Sunderland, C. (2011b). Neck cooling and running performance in the heat: Single versus repeated application. Medicine and Science in Sports and Exercise, 43(12), 2388-2395. doi: 10.1249/ MSS.0b013e318222ef72

Watanuki, S. (1993). Effects of head cooling on cardiovascular and body temperature responses during submaximal exercise. The Annals of physiological Anthropology, 12(6), 327-333.

Watkins, S. L., Castle, P., Mauger, A. R., Sculthorpe, N., Fitch, N., Aldous, J., . . . Taylor, L. (2014). The effect of different environmental conditions on the decisionmaking performance of soccer goal line officials. Research in Sports Medicine, 22(4), 425-437. doi: 10.1080/15438627.2014.948624 